\title{
Appendix 1: Statistics
}

\section{Experiment 1: Quantifier Scope}

\section{Surface scope descriptions}

Random effects

\begin{tabular}{ccc}
\hline \hline groups & variance & sd \\
\hline \hline subjects & 1.29 & 1.14 \\
\hline \hline items & 0.49 & 0.7 \\
\hline \hline
\end{tabular}

Fixed effects:

\begin{tabular}{cccc}
\hline \hline measure & estimate & z-value & p-value \\
\hline \hline (intercept) & $3.86(0.61)$ & 6.36 & $2.08 \mathrm{e}-10 * * *$ \\
\hline \hline unaccusative & $0.04(0.64)$ & 0.07 & 0.95 \\
\hline \hline unergative & $-1.09(0.59)$ & -1.86 & 0.06 \\
\hline \hline
\end{tabular}

$* * *=p \leq 0.001$

\section{Inverse scope descriptions}

Random effects

\begin{tabular}{ccc}
\hline \hline groups & variance & sd \\
\hline \hline subjects & 1.52 & 1.23 \\
\hline \hline items & 0.86 & 0.93 \\
\hline \hline
\end{tabular}

Fixed effects:

\begin{tabular}{cccc}
\hline \hline measure & estimate & z-value & p-value \\
\hline \hline (intercept) & $-3.82(0.57)$ & -6.69 & $2.20 \mathrm{e}-11 * * *$ \\
\hline \hline unaccusative & $4.93(0.72)$ & 6.87 & $6.26 \mathrm{e}-12 * * *$ \\
\hline \hline unergative & $0.43(0.67)$ & 0.65 & 0.52 \\
\hline \hline
\end{tabular}

$* * *=p \leq 0.001$ 


\section{Experiment 2: FNQ-licensing}

Random effects

\begin{tabular}{ccc}
\hline \hline groups & variance & sd \\
\hline \hline subjects & 0.02 & 0.14 \\
\hline \hline items & 0.1 & 0.32 \\
\hline \hline residual & 0.27 & 0.52 \\
\hline \hline
\end{tabular}

Fixed effects:

\begin{tabular}{ccccc}
\hline \hline measure & estimate & df & t-value & p-value \\
\hline \hline (intercept) & $0.5(0.13)$ & 83.08 & 3.8 & $0.0002 * * *$ \\
\hline \hline FNQ & $-0.16(0.18)$ & 77.54 & -0.86 & 0.4 \\
\hline \hline class 2 & $0.09(0.18)$ & 77.54 & 0.52 & 0.61 \\
\hline \hline class 3 & $0.21(0.18)$ & 77.54 & 1.17 & 0.25 \\
\hline \hline class 4 & $-0.04(0.18)$ & 77.54 & -0.216 & 0.83 \\
\hline class 5 & $-0.07(0.18)$ & 77.54 & -0.4 & 0.69 \\
\hline \hline FNQ x class 2 & $0.14(0.26)$ & 77.54 & 0.55 & 0.58 \\
\hline \hline FNQ x class 3 & $0.17(0.26)$ & 77.54 & 0.66 & 0.51 \\
\hline \hline FNQ x class 4 & $-0.68(0.26)$ & 77.54 & -2.64 & $0.01 *$ \\
\hline \hline
\end{tabular}

$* * *=p \leq 0.001, *=p \leq 0.05$

Experiment 3: FNQ-licensing with animacy and telicity

\section{Animacy (unaccusatives)}

Random effects

\begin{tabular}{ccc}
\hline \hline groups & variance & sd \\
\hline \hline subjects & 0.01 & 0.08 \\
\hline \hline items & 0.01 & 0.09 \\
\hline \hline residual & 0.05 & 0.22 \\
\hline
\end{tabular}

Fixed effects:

\begin{tabular}{ccccc}
\hline \hline measure & estimate & $\mathrm{df}$ & t-value & p-value \\
\hline \hline (intercept) & $0.13(0.04)$ & 54.34 & 3.46 & $0.001^{* *}$ \\
\hline \hline FNQ & $-0.001(0.05)$ & 44.19 & -0.03 & 0.97 \\
\hline \hline animacy & $0.11(0.05)$ & 44.19 & 2.267 & $0.03^{*}$ \\
\hline \hline FNQ x animacy & $-0.03(0.07)$ & 44.19 & -0.49 & 0.63 \\
\hline
\end{tabular}

$* *=p \leq 0.01, *=p \leq 0.05$ 


\section{Telicity (unergatives)}

Random effects

\begin{tabular}{ccc}
\hline \hline groups & variance & sd \\
\hline \hline subjects & 0.003 & 0.05 \\
\hline \hline items & 0.01 & 0.08 \\
\hline \hline residual & 0.04 & 0.21 \\
\hline \hline
\end{tabular}

Fixed effects:

\begin{tabular}{ccccc}
\hline \hline measure & estimate & df & t-value & p-value \\
\hline \hline (intercept) & $0.16(0.04)$ & 33.03 & 3.85 & $0.0005^{* * *}$ \\
\hline \hline FNQ & $-0.14(0.06)$ & 31.18 & -2.4 & $0.02 *$ \\
\hline \hline telicity & $-0.03(0.06)$ & 31.18 & -0.48 & 0.64 \\
\hline \hline FNQ x telicity & $0.09(0.08)$ & 31.18 & 1.19 & 0.24 \\
\hline \hline
\end{tabular}

$* * *=p \leq 0.001, *=p \leq 0.05$

Telicity (class 4)

Random effects

\begin{tabular}{ccc}
\hline \hline groups & variance & sd \\
\hline \hline subjects & 0.002 & 0.04 \\
\hline \hline items & 0.00 & 0.00 \\
\hline residual & 0.04 & 0.21 \\
\hline \hline
\end{tabular}

Fixed effects:

\begin{tabular}{ccccc}
\hline \hline measure & estimate & $\mathrm{df}$ & t-value & $\mathrm{p}$-value \\
\hline \hline (intercept) & $0.19(0.04)$ & 119.49 & 4.77 & $5.18 \mathrm{e}-06^{* * *}$ \\
\hline \hline FNQ & $-0.05(0.05)$ & 90.00 & -0.9 & 0.37 \\
\hline \hline telicity & $-0.07(0.05)$ & 90.00 & -1.35 & 0.18 \\
\hline \hline FNQ x telicity & $-0.03(0.08)$ & 90.00 & -0.37 & 0.71 \\
\hline \hline
\end{tabular}

$* * *=p \leq 0.001$

Telicity (class 5)

Random effects

\begin{tabular}{ccc}
\hline \hline groups & variance & sd \\
\hline \hline subjects & 0.004 & 0.06 \\
\hline \hline items & 0.003 & 0.05 \\
\hline \hline residual & 0.05 & 0.21 \\
\hline \hline
\end{tabular}


Fixed effects:

\begin{tabular}{ccccc}
\hline \hline measure & estimate & $\mathrm{df}$ & t-value & p-value \\
\hline \hline (intercept) & $0.13(0.05)$ & 15.11 & 2.63 & $0.02^{*}$ \\
\hline \hline FNQ & $-0.22(0.07)$ & 12.83 & -3.3 & $0.006^{* *}$ \\
\hline \hline telicity & $0.02(0.07)$ & 12.83 & 0.29 & 0.78 \\
\hline \hline FNQ x telicity & $0.21(0.09)$ & 12.83 & 2.27 & $0.04^{*}$ \\
\hline \hline
\end{tabular}

$* *=p \leq 0.01, *=p \leq 0.05$ 